\title{
The Possibility of Weak V-DE-(NP)-A Constructions in Chinese
}

\author{
Xiaowen Zhang \\ Huaiyin Institute of Technology, Huaian, Jiangsu, China
}

\begin{abstract}
According to Washio's (1997) strong and weak resultative analysis, Chinese resultative V-A-(NP) compounds allow both strong and weak resultative constructions while Chinese V-DE-(NP)-A constructions allow only strong resultative constructions, i.e., weak V-DE-(NP)-A constructions are not accepted in these constructions when the result predicate is a stage-level predicate $+l e$. However, it can be found that these ungrammatical weak V-DE-(NP)-A constructions are possible to be grammatical in Chinese when the result predicate is an individual-level predicate or is modified by a degree word. A natural question to ask here is why so. In the paper, I will suggest a reason for it in terms of the function of DE and the aspect marker $l e$ in Chinese resultative constructions and the syntactic structures of V-DE-(NP)-A constructions.
\end{abstract}

Index Terms - Chinese resultative constructions, strong resultatives, weak resultatives

\section{INTRODUCTION}

\section{A. Resultative Constructions in English and Chinese}

In resultative constructions, besides the main verb $(\mathrm{V})$, there is a secondary predicate called the result phrase (XP), including an adjective phrase (AP) or a preposition phrase (PP) or even a verb (V), that is, V-V compounds in Chinese. For example:

(1) a. The gardener watered the flowers flat.

b. John broke the vase into pieces.
c. Zhangsan sha-si le Lisi.
Zhangsan kill-dead ASP Lisi
'Zhangsan killed Lisi dead.'

(1a) means that the flowers became flat because of gardener's watering it, and (1b) means that the vase went to pieces because John broke it. (1c) means that Lisi was dead because Zhangsan killed him. Depending on these sentences, it can be observed that they mainly describe a state which results from the action rather than simply describe the action typically expressed by the main verb.

Resultative constructions in Chinese were first proposed by Ding (1961). As he points out, it is composed of two parts - the verb and the complement, in which the latter supplements the former and indicates the result of the action demonstrated by the former part. In other words, the primary one denotes the event of an action and the secondary one denotes the event of a consequence or a state accompanied by the action. As Li (1998) noted, a resultative construction illustrates a superevent constituted by two subevents, the cause and the result, as demonstrated in (2), we refer to such a construction as 'dong bu (verb-complement)' construction in Chinese.

(2)

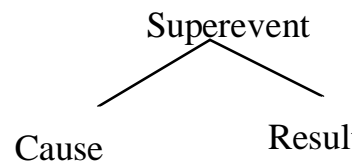

$$
\text { (Li, 1998: 293) }
$$

And sometimes the auxiliary DE exists between the main verb and the result phrase, as highlighted by Li (2003: 7). He claims that a resultative construction can be divided into types with or without " $d e$ " from the viewpoint of form, that is, resultative V-C(omplemet)-(NP) compounds and V-DE-(NP)-C constructions. Zhang (2019) use a tree diagram to make clear of the relations between two types of Chinese resultative constructions, as shown in (3). 
(3)

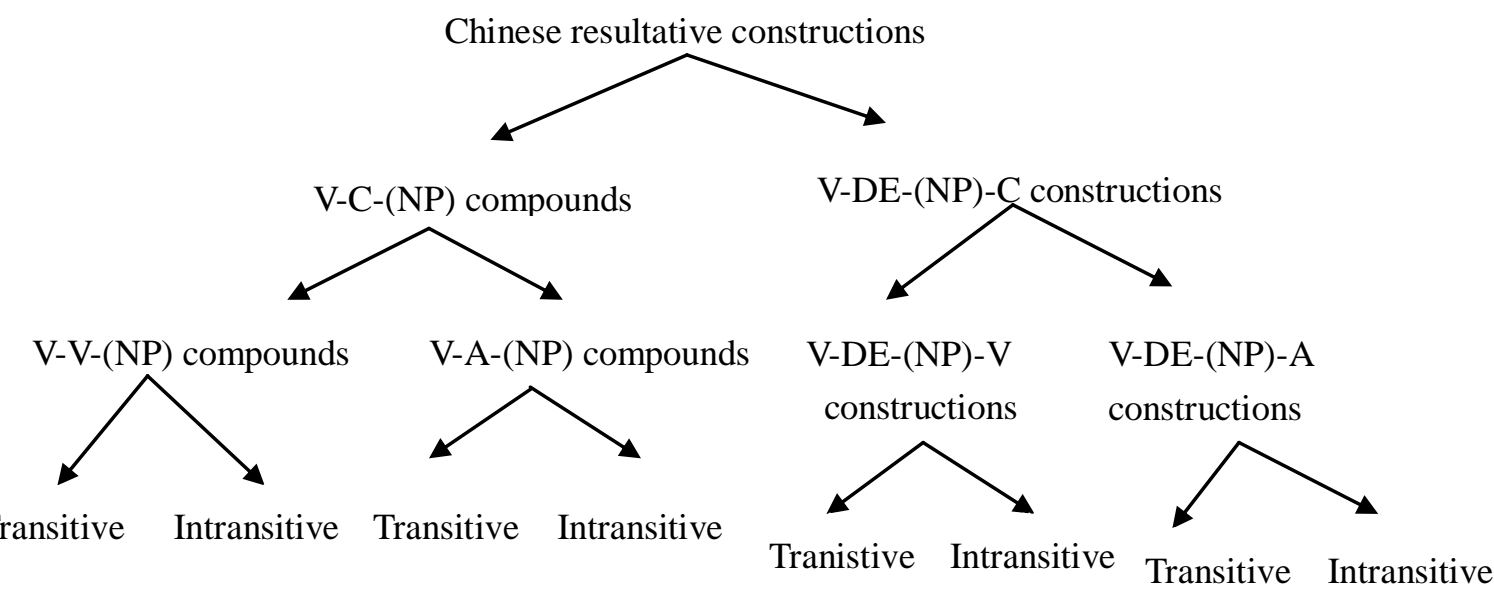

A resultative V-V-(NP) compound in Chinese is constructed by compounding two verbal morphemes, the first morpheme indicating a causing event, marked by $\mathrm{V}_{1}$ and the second one indicating the resulting event, marked by $\mathrm{V}_{2}$. Different from the resultative $\mathrm{V}-\mathrm{V}-(\mathrm{NP})$ compound in Japanese, $\mathrm{V}_{1}$ is the head of the compound in Chinese. The $\mathrm{V}$-DE-(NP)- $\mathrm{V}$ construction is also constructed by two verbal morphemes, with $\mathrm{V}_{1}$ denoting the cause and $\mathrm{V}_{2}$ the result, but $V_{1}$ and $V_{2}$ are separated by DE. Different from the couple of the resultative V-V-(NP) compound and the $\mathrm{V}$-DE-(NP)-V construction, the result predicate is an adjective in the resultaive $\mathrm{V}-\mathrm{A}-(\mathrm{NP})$ compound and the V-DE-(NP)-A construction. Based on the diagram in (3), the specific divisions of Chinese resultative constructions and some specific examples are shown in (4).

(4) the V-V-(NP) compound
a. $\mathrm{NP}_{1} \quad \mathrm{~V}_{1}-\mathrm{V}_{2} \quad$ (intransitive)
Mary qi-si le.
Mary anger-die ASP
'Mary angered herself dead.'
b. NP1 V1-V2 NP2 (transitive)
Wusong da-si le laohu.
Wusong hit-die ASP tiger
'Wusong hit the tiger dead.'
the V-A-(NP) compound
c. $\mathrm{NP}_{1} \quad \mathrm{~V}-\mathrm{A} \quad$ (intransitive)
Taotao ku-lei le.
Taotao cry-tired ASP
'Taotao cried himself tired.'
d. $\mathrm{NP}_{1} \quad \mathrm{~V}-\mathrm{A} \quad \mathrm{NP} 2$ (transitive)
John kan-lan le nabenshu.
John see-broken ASP that book
'John read that book so frequently that it was broken.'
the V-DE-(NP)- $V$ construction
e. $\mathrm{NP}_{1} \quad \mathrm{~V}_{1}-\mathrm{DE}-\mathrm{V}_{2} \quad$ (intransitive)
John qi de yaoyaqiechi.
John anger DE gnash the teeth
'John gnashed her teeth with rage.'
f. NP1 V1-DE NP2 V2 (transitive)
John qi de wo yaoyaqiechi.
John anger DE me gnash the teeth
'John angered me gnashing my teeth.'
the V-DE-(NP)-A construction
g. NP1 V-DE-A (intransitive)
Zhangsan kan de hen lei.
Zhangsan read DE very tired
'Zhangsan read very tired.'
h. NP1 V-DE-A NP2 (intransitive)
John kan de naben shu po le.
John read DE That CL book broken ASP
'John read that book till that book became broken.'


The resultative V-V-(NP) compound is constructed by compounding two verbal morphemes, as in (4b), in which $\mathrm{V}_{1}$ $d a$ 'hit' indicates a causing event and $\mathrm{V}_{2} s i$ 'dead' indicating the resulting event. The resultative $\mathrm{V}$-A-(NP) compound is constructed by compounding two morphemes: a verbal one and an adjectival one, as in (4d), in which the verb kan 'see' indicates a causing event and the adjective lan 'broken' indicating the resulting event. The V-DE-(NP)-V construction is also constructed by two verbal morphemes, as in (4f), with $\mathrm{V}_{1}$ qi 'anger' denoting the cause and $\mathrm{V}_{2}$ yaoyaqiechi 'gnash the teeth' denoting the result. The V-DE-(NP)-A construction is constructed by a verbal morpheme kan 'see' and an adjectival one po 'broken', as in (4h). In (4f and $\mathrm{h}$ ), $\mathrm{V}_{1}$ and $\mathrm{V}_{2} / \mathrm{A}$ are separated by $\mathrm{DE}$ and the postverbal object wo 'myself' and nabenshu 'that book', whereas without the postverbal object, $\mathrm{V}_{1}$ and $\mathrm{V}_{2} / \mathrm{A}$ are only separated by $\mathrm{DE}$, as in (4e and $\mathrm{g})$.

\section{B. Washio's (1997) Strong and Weak Resultatives}

Washio (1997) claims that resultative constructions can be classified as strong and weak resultatives at least in terms of the meaning of the main predicate. Strong resultatives means that the meaning of the verb and the meaning of the adjective are completely independent of each other. In strong resultatives, it is impossible to predict what kind of state the patient comes to be from the semantics of the verb in as the result of the action named by the verb. For example:

(5) a. Mary watered the flowers flat.

b. John danced his feet sore.

In (5a), the verb water doesn't imply any state of the result phrase that might result from the action it names, i.e. flat. In (5b), the verb dance is unergative, so it cannot contain anything like the notion sore denoted by the adjective that is predicated of the 'fake object' in its lexical semantics. So examples in (5) are strong resultatives.

Washio (1997) points out that weak resultatives refers to those ones the meaning of the verb entails the meaning of the adjective, that is, in weak resultatives, if the states of the patients change, verbs will imply that they would change in certain fixed directions to reach the final states. For example: (cited from Washio (1997))

(6) a. I dyed the dress pink.

b. Mark froze the ice cream solid.

c. John painted the wall white.

In (6a), although the verb $d y e$ doesn't specifically contain the notion pink, it clearly contains the notion 'color'. If so, the adjective pink cannot be completely independent of the verb dye. In (6b), the notion solid is closely related to the meaning of freeze. In $(6 \mathrm{c})$, the meaning of the verb paint entails the change of the wall's color; the adjectival result phrase white denotes the result. It can thus be said that examples in (6) are weak resultatives. Therefore, it can be said that both strong and weak resultatives are allowed in English.

\section{Strong and Weak Resultatives in Chinese Resultative Constructions}

There are two main types of Chinese resultative constructions focused in the paper, that is, resultative V-A-(NP) compounds and V-DE-(NP)-A constructions. In section 2, strong and weak resultatives are examined in Chinese two types of resultative constructions, respectively.

\section{A. Strong and Weak Resultatives in Resultative V-A-(NP) Compounds}

Let us consider examples in (7) and (8) at first.

(7) Strong resultatives in V-A-(NP) compounds
a. Mary $k u$-shi le shoupa.
Mary cry-wet ASP handkerchief
'Mary cried to a extend as a result the handkerchief got wet.'
b. Lisi jiao-ta le hua.
Lisi water-flat ASP flowers
'Lisi watered the flowers flat.'

(8) Weak resultatives in V-A-(NP) compounds
a. Zhangsan tu-bai-le qiang.
Zhangsan paint-white-ASP wall
'Zhangsan painted the wall white.'
b. Mary ran-hong-le qunzi.
Mary dye-red-ASP the dress
'Mary dyed the dress red.'

$(7 \mathrm{a}, \mathrm{b})$ are strong resultatives, which shows that they are allowed in Chinese resultative V-A-(NP) compounds. (8a, b) are weak resultatives, which shows that they are allowed in resultative V-A-(NP) compounds. Based on examples in (7-8), it can be said that like English, both strong and weak resultatives are allowed in Chinese resultative V-A-(NP) compounds.

After many examples of Chinese resultative V-A-(NP) compounds are allowed, which shows that they are productive in Chinese, for example, some strong resultatives are grammatical in Chinese, but they are ungrammatical in English, as shown in (9). 
(9) a.Zhangsan chi-huai le duzi.

Zhangsan eat-bad ASP stomach

'Zhangsan has eaten (something bad or too much, as a result he has an) upset stomach.

*Zhangsan ate his stomach bad.

b. He ti-po le qiuxie. (Cheng and Huang (1994))

He kick-break ASP sneaker

'He kicked so much that the sneakers were broken.'

*He kicked his sneakers broken.'

The examples in (9) cannot be acceptable in English while they are grammatical in Chinese. In (9b), as Cheng and Huang (1994) points out, qiuxie 'sneaker' is not the actual object of V $t i$ 'kick'. What John kicked may be a football. In this case, $\mathrm{V} t i$ ' kick' thus functions like an intransitive verb. If so, it can be said that Chinese allows some wider range of strong resultatives, the reason of which still remains a mystery in the contemporary Chinese linguistic.

\section{B. Only Strong Resultatives in V-DE-(NP)-A Constructions}

After strong and weak resultatives are examined in Chinese V-DE-(NP)-A constructions, it can be found that only strong ones are allowed in V-DE-(NP)-A constructions, without weak ones, as shown in (10).
$\begin{array}{rlrrr}\text { (10) a. Mary } & k u \text { de } & \text { shoupa shi le. } \\ \text { Mary cry DE shoupa wet ASP }\end{array}$
'Mary cried to a extend as a result that the handkerchief got wet.'
b. Zhangsan jiao de hua ta le.
Zhangsan water DE the flower flat ASP
'Zhangsan watered the flower flat.'
c. *Wo tu de qiang bai le.
I painted DE the wall white ASP
'I painted the wall white.'
d. *Lisi dong de bingqilin ying le.
Lisi freeze DE the ice cream solid ASP
'He froze the ice cream solid.'

In $(10 \mathrm{a}, \mathrm{b})$, the adjective $s h i$ 'wet' and bian 'flat' are not implied by the verb $k u$ 'cry' and jiao 'water', namely, they are strong resultatives. The examples in $(10 \mathrm{a}, \mathrm{b})$ indicate that strong resultatives are acceptable in V-DE-(NP)-A constructions. In $(10 \mathrm{c}, \mathrm{d})$, the adjectives bai 'white' and ying 'solid' can be implied by the verbs $t u$ 'paint' and dong 'freeze', but the sentences are ungrammatical, which shows that weak ones are unacceptable in V-DE-(NP)-A constructions. By contrast, both strong and weak resultatives are acceptable in Chinese resultative V-A-(NP) compounds, so this sharp difference must be noted.

More examples of ungrammatical weak resultatives in Chinese V-DE-(NP)-A constructions are shown in (11).

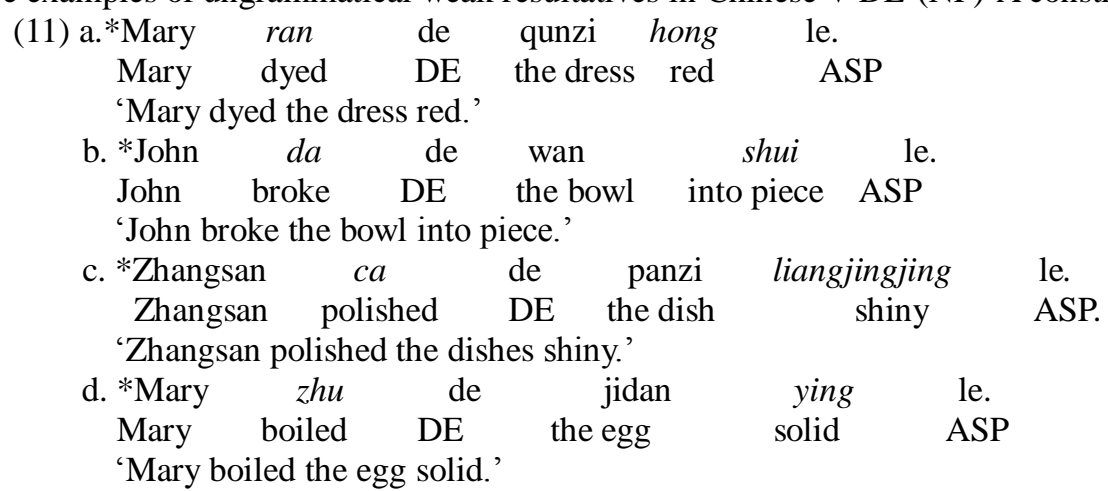

The examples in (10c-d) and (11) show that weak resultatives are unacceptable in Chinese V-DE-(NP)-A constructions, but interestingly, these ungrammatical weak resultatives can be allowed in Chinese when the result predicate is an individual-level predicate (hereafter ILP) or the result predicate (a stage-level predicate (hereafter SLP)) is modified by a degree word. Why so? In section 3, I will suggest a reason for this question.

\section{The Possibility of Grammatical WeAK V-DE-(NP)-A CONSTRUCTIONS In Chinese}

After inquiring many Chinese native speakers, I pay attention to an interesting phenomenon that when the result predicate is a combination of a SLP + the aspect marker le, weak V-DE-(NP)-A constructions are ungrammatical, whereas when the result predicate is an ILP or it is modified by a degree word, ungrammatical weak V-DE-(NP)-A constructions become grammatical, as shown in (12).
(12) a. *John tu de qiang bai le.
John paint DE wall white ASP
'John painted the wall white.' 


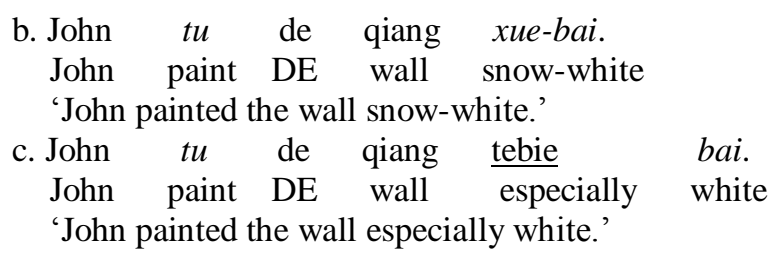

In (12a), when the result predicate is the combination of SLP + le, weak V-DE-(NP)-A constructions in Chinese are ungrammatical, whereas when the result predicate is an ILP or is modified by a degree word, these ungrammatical weak ones become grammatical in Chinese. Why so? Considering the ungrammatical weak V-DE-(NP)-A construction deeply, it can be realized that this question may be related to the function of the time-phased auxiliary le in Chinese, because in the case of the result predicate as a ILP or is modified by a degree word, le disappears, that is, le cannot be combined with an ILP or the combination of the degree word + SLP, as shown in (13).
(13) a. *John tu de qiang snow-bai le. John paint DE wall snow-white ASP 'John painted the wall snow-white.'

In (13a), the ILP xue-bai 'snow-white' is an ILP, corresponding to dynamic adjectives, proposed by Zhang (1995). Based on the definition of the dynamic adjective, it cannot be followed by the time-phased auxiliary le, which leads to (13a) ungrammatical. In (13b), when the degree word hen 'very' modifies the combination of SLP + le 'bai-le', it can only modify the SLP bai 'white' but not le. Thus, when the degree word appears, the time-phased auxiliary le must be deleted. If not so, the sentence will be ungrammatical, as shown in (13b).

Before discussing the question why ungrammatical weak V-DE-(NP)-A constructions become grammatical when the result predicate is an ILP or is modified by a degree word, it is necessary to clarify the definition s of SLP and ILP in English and dynamic adjectives and static adjectives at first.

\section{A. Stage-level and Individual-level Predicates in English}

Carlson (1977) argues that predicates can be classified into two natural classes: stage-level predicates (SLPs) and individual-level predicates (ILPs) in English. At first, let us consider two pairs of examples from Carlson (1977), as shown in (14) and (15).

(14) a. He saw the president naked.

b. *He saw the president intelligent.

(15) a.Mark saw Mary talk to Bill.

b. *Mark saw Mary know Bill.

Carlson (1977) proposes that the predicates like intelligent in (14b) or to know Bill in (15b) are unacceptable in these constructions, because these predicates are 'individual-level predicates' that usually express permanent and essential properties. The predicates that are acceptable, like naked in (14a), talk to Bill in (15a), which are called 'stage-level predicates', tending to denote transitory and accidental properties.

\section{B. Kratzer's (1995) Distinguishment of SLPs with ILPs by Event Argument}

The event argument, as introduced by Dividson (1967), has not only been used for the analysis of action sentences, but also for a variety of purposes in the recent literature. According to his analysis, a sentence like (16) below states the existence of a past event which is a purchase of those books by Mary, and which takes place in the book store.

(16) Mary purchased those books in the book store.

In (16), the verb purchase is a three-place predicate. In addition to an event argument, it has an argument denoting the agent Mary and the other one denoting the theme, the object of the purchase. Namely, the subject and the direct object correspond to arguments of the main predicate, while the locative argument the book store is introduced by a secondary predicate, the preposition in. Davidson suggests that verbs describe events or states with the logician's way of thinking about verb denotations is by adopting the logician's view and add an extra argument, the event argument. As a result, verb meanings determine a verb's arguments while they also characterize a set of events or states.

Based on Dividson's (1967) event argument analysis, Kratzer (1995) argues that the difference between SLPs and ILPs can be captured in terms of presence and absence of the event argument. She illustrates this claim with the help of the following examples, for instance, SLPs like hit contain an event argument ("location" in her terms) as in (17a), and ILPs like know lack it as in (17b).

(17) a. hit < location, agent, theme >

b. know < experience, theme >

Specifically, she believes that SLPs are distinct with ILPs in that they contain an explicit reference to a place and a time. For example:

(18) a. John was tired / nervous in the car.

(David 2007)

b. *John was intelligent / a linguist in the car. 
(19) a. John was drunk yesterday/last month/a year ago. (Carlson 1977)

b. *John was tall yesterday/last month/a year ago.

In (18a), stage-level predicates, like tired, nervous can be combined with the locative modifier in the car, while individual-level predicates, like intelligent, a linguist don't seem to accept a location in (18b). In (19a), drunk is a stage-level predicate, which can be combined with the temporal adverbials, like yesterday, last month and a year ago, while tall is an individual-level predicate and cannot be combined with these adverbials as in (19b).

\section{SLPs and ILPs in Chinese Adjectives}

According to Zhu (1982), in addition to a few unaccusative verbs that represent changes of state, the result predicates are basically adjectives in Chinese resultative constructions. Thus, the study of Chinese adjectives becomes very important in Chinese contemporary linguistics.

Zhang (1995) refines the result predicates in Chinese resultative constructions and proposes that adjectives in Chinese can be divided into 'dynamic adjectives' and 'static adjectives' and he also analyzes the semantic structure of adjectives in details. Here, based on Carlson (1977) and Kratzer (1995), I assert that dynamic adjectives in Chinese resultatives are parallel to SLPs, which have temporary or accidental properties, while static adjectives are parallel to ILPs in Chinese resultatives, which have permanent or inherent properties.

According to Zhang (1995), most of dynamic adjectives (SLPs) in Chinese adjectives are monosyllable and their time structures are heterogeneous, which means that SLPs have intrinsic natural start and end points. Thus, SLPs can appear together with some time-phased auxiliaries "le" or "zhuo" in syntax of Chinese. For example:

(20) Dynamic adjectives (SLPs): bai (white), hei (black), hong (red), chang (long), duan (short), da(large), xiao (small), gao (high), di (low), chen (heavy), chou (stinky), cu (thick), fei (fat), gan (dry), kong (empty), lan (bad), shui (broken), shi (wet), yuan (round), teng (sore), ying (hard), etc.

By contrast, most of static adjectives (ILPs) in Chinese are double syllables and their time structures are homogeneous, which lack intrinsic time start and end points. And ILPs cannot appear together with some time-phased auxiliaries "le" or "zhuo" in syntax of Chinese. For example:

(21) Static adjectives (ILPs): duanzan (short), manchang (long), haofang (unrestrained), benzhong (unwieldy), anggui (expensive), feida (hypertrophy), huanle (joy), jianxin (hardships), meimiao (wonderful), minggui (luxurious), ningjing (quiet), qinrao (hardworking), qingwei (slight), xiangjin (detailed), youxiu (excellent), zhengdang (legitimate), zhuangli (magnificent), xuebai (snow-white), etc.

Depending on the definitions of dynamic and static adjectives, Zhang (1995) gives the following examples to distinguish SLPs with ILPs in Chinese adjectives, as shown in (22).

(22) a. Zhangsan tu-bai paint-white ASP the wall
Zhangsan le qiang.
'Zhangsan painted the wall white.'
b. *Zhangsan tu xue-bai le qiang.
Zhangsan paint snow-white ASP the wall
'Zhangsan painted the wall snow-white.'

The result predicate bai 'white' in (22a) is a SLP, which represents the change of temporary state of the thing, and it can appear together with the time-phased auxiliary le. In (22b), however, xuebai 'snow-white' is an ILP in Chinese, which indicates the constant and static state of the thing, and it cannot appear together with the time-phased auxiliary le, so (22b) is ungrammatical.

Moreover, depending on Kratzer's (1995) event argument analysis, only SLPs have the event argument in their argument structure and ILPs lack it in Chinese resultatives, as shown in (23).
(23) a. Yi xiao shi nei, qiang bei tu-bai
In an hour, wall le.
'The wall was painted white in an hour.'
b. * Yi xiao shi nei, qiang bei tu xuebai.
In an hour, wall was paint snow-white
'The wall was painted snow-white in an houe.'

In (23a), bai 'white' is a dynamic adjective, i.e. a SLP, which can contain an event argument yi xiao shi nei 'in an hour', while in (23b), xue-bai 'snow-white' is a static adjective, i.e. an ILP, in which an event argument, like xiao shi $n e i$ 'in an hour' is not allowed. Thus, (23b) is unacceptable. Until now, the definitions and distinguishes of SLP and ILP in English and Chinese are introduced, and the focused question will be accounted for in the following subsections which is related to the functions of $\mathrm{DE}$ and the aspect marker $L e$ in Chinese resultative constructions.

\section{The Function of the Aspect Marker Le in Chinese}

Chinese has a rich aspectual system. Aspect markers refer to the elements contributing to the outer viewpoint of a verbal event, such as le (了), guo (過), zhe (着) in Chinese. These three aspect markers show the different telicity information on the verb. As a perfective aspect marker, le expresses the completion of an action, and guo indicates more on the past experience of an action or state. Zhe is taken as a durative aspect marker which indicates an imperfective event, as shown in (24). 


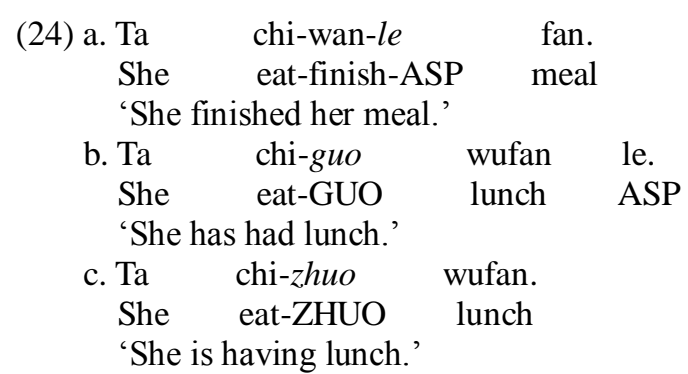

In (24a), the compound chi-wan 'eat-finish' already denotes the result, which means that she finished her meal because of her eating action. Le imposes an endpoint to the event, emphasizing the completion of the event. In (24b), guo, as a perfective verbal suffix, indicates the whole action as a past experience with absolute completion. (24b) thus means that she experienced the action of eating her lunch and as a result this action has been completely finished. In (24c), zhe, as a verbal suffix, denotes the continuous state of an imperfective event, and (24c) means that she is eating her lunch and this action is not over. Here I only simply introduce the function of aspect marker guo and zhuo. In the thesis, I focus exclusively on the aspect marker $l e$.

The perfective marker $l e$ can highlight a change of state, denoting the boundaries of an event either at the starting point or at the endpoint. If the event itself is telic, le denotes the endpoint of the event, as in (24a). When le follows a SLP, it denotes the starting point of change of a temporary state expressed by the SLP, as in (25). In a word, le co-occurs with verbs or adjectives that involve a change of state, where the event has reached an end in the case of co-occurring with verbs, while the event is being initiated in the case of co-occurring with adjectives.
(25) Qiang bai le.
Wall white ASP
'The wall became white.'

In (25), bai 'white' is a SLP which denotes the temporary state. When the SLP bai 'white' is followed by le, the meaning of the sentence is changed and le here denotes the starting point of change.

We can say that $l e$ denotes the result semantically and it indicates a change of state -- either it can end a previous state or can initiate another state. When le follows the SLP, bai 'white' denoting the temporary state will begin to change. Without the appearance of le, the sentence qiang bai 'the wall white' just describes the temporary state of the wall and no change happens.

\section{E. The Function of DE in Chinese Resultative Constructions}

As to the function of DE in Chinese resultative V-DE-(NP)-A constructions, based on Folli's (2001) three-layer analysis, Lin (2003) proposes that DE can be indicated as one of layers, a Process. Folli (2001) proposes that in addition to the causing and resultative events, there is an intermediate event denoting a process in English resultative constructions. It thus can be said that a resultative construction has three semantic layers, that is, the cause, the process and the result, and Folli (2001) shows his claim in syntactic structure, as shown in (26).

(26)

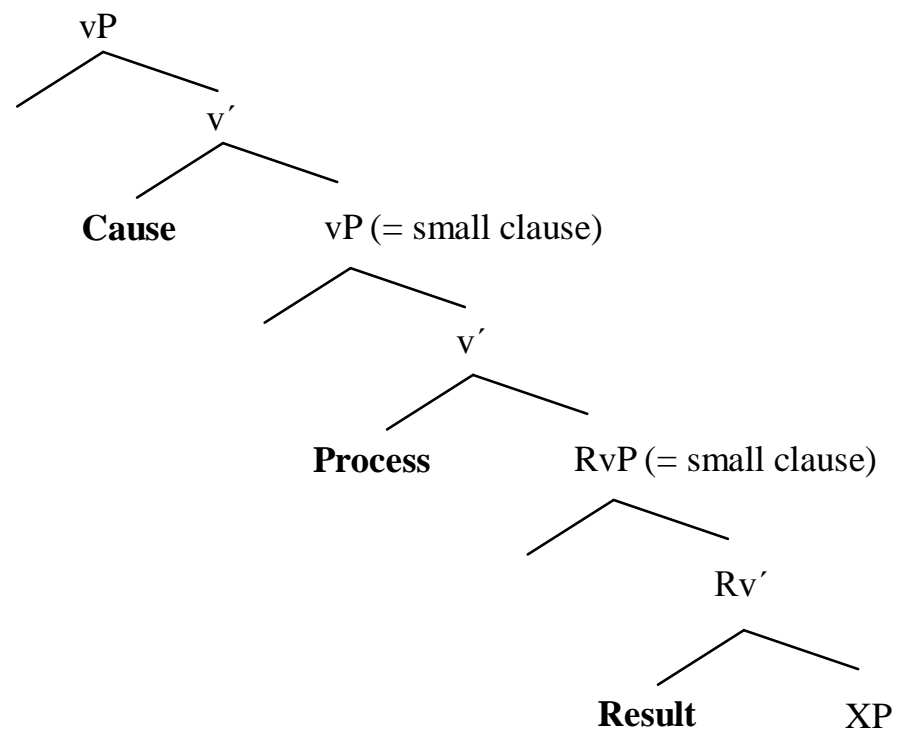

Folli provides a piece of evidence to show that the process should be separated from the cause and the result, as in (27).

(27) John rolled the ball to the wall very fast. 
a. John very quickly rolled the ball so that it reaches the wall. (cause)

b. John pushed the ball so that it rolls to the wall very fast. (process)

c. John pushed the ball to the wall as a result it rolls very fast. (result)

The sentence (27) can be interpreted from three aspects, i.e., cause, process and result. Folli argues the adverbial word (fast, quickly) can modify different parts of a complex event. When it modifies the cause, (27a) means that John's action of causing the ball to roll was very fast. When it modifies the rolling process, $(27 \mathrm{~b})$ means that the action of the ball rolling was very fast before reaching the wall. When it modifies the result, $(27 \mathrm{c})$ means that John's action of rolling the ball to the wall made the ball roll very fast. Folli (2001) points out that the cause, the process, and the result of the action can be modified respectively, so it can be said that these three subevents should be separated in the syntactic structure, as in (26).

Lin (2003) proposes that DE can be indicated as a process head in Chinese V-DE-(NP)-A constructions based on Folli's three-layer analysis. Take an example from Lin (2003) in (28).

(28) He qi de toupi fa ma.

He angry DE scalp get numb

'He was angry to the point that my scalp got numb.'

The sentence (28) means that I was angry to the extent that my scalp became numb. Thus, here DE can indicate a process in which anger leads to the physical (scalp-becoming-numb) reaction.

Lin's analysis of DE as a process head is reasonable, I think, because Hein \& Kuteva (2002) propose that the English verb get or obtain often grammaticalizes to mean the process of change cross linguistically. And DE in Chinese actually means 'get' or 'obtain', as in (29).

(29) wo shuxue de le 100 fen.

I mathematics got ASP 100 marks

'I got 100 marks in the mathematics examination.'

The sentence (29) further indicates that DE can be indicated as a Process in Chinese resultative constructions based on Folli's three-layer system.

\section{F. The Similarities between the Functions of DE and Le in Chinese Resultatives}

Folli and Lin's proposal has been adopted that DE can serve as the process head in the syntactic structure of the V-DE-(NP)-A construction in Chinese, that is, DE can be indicated as a process in Chinese resultative constructions. Repeated Folli's three-layer syntactic structure in resultative constructions here as in (30).

(30)
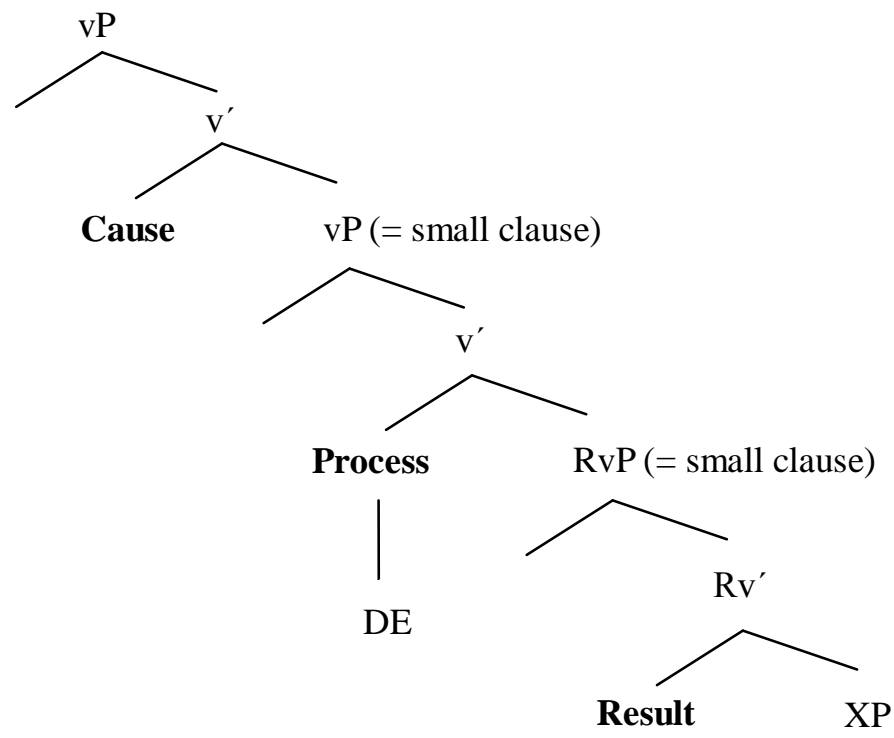

According to Folli (2001), the process and the resultative event can be seen as small clauses embedded within the causative event, as in (30). Here Lin (2003) focuses on the resultative function of DE, considering it as the process head in Chinese resultative constructions. The fact that the process head is filled by DE in Chinese resultative constructions keeps the resultative head $\left(\mathrm{V}_{2}\right)$ from moving upwards as in the resultative V-V-(NP) compound. Thus, DE can be seen as the intermediate between the cause and the result, as shown in (30).

Folli (2001) proposes that as a process, it can be considered as the result of $\mathrm{V}_{\text {cause }}\left(\mathrm{V}_{1}\right)$, and further leading to $\mathrm{V}_{\text {result }}$ $\left(\mathrm{V}_{2}\right)$. Namely, I interprete her view as DE can denote the endpoint of the change on the main verb expressing Cause and it can also denote the starting point of the change of Result. For example:
(31) Mary
$k u$
de shoupa
shi-le.
Mary
DE the handkerchief
wet-ASP 
'Mary cried as a result that the handkerchief got wet.'

In (31), DE indicates a process between her crying and the result that the handkerchief got wet. Here DE has no lexical meaning but can be indicated as a functional word, which means the completion of the action denoted by $\mathrm{V}_{1} k u$ 'cry' and the beginning of the action denoted by $\mathrm{V}_{2}$ shi 'wet'.

The function of the aspect marker $L e$ has been accounted for in section 3.4, that is, le can also indicate a change of state -- either denotes the endpoint of change on the main verb $\mathrm{V}_{1}$ or the start point of change on the result predicate $\mathrm{V}_{2}$. Associating the function of DE with the aspect marker le here, it can be found that their functions are similar, because both $\mathrm{DE}$ and $L e$ can indicate a change of state and DE denotes the endpoint of change on the main verb expressing Cause and the start point of change on Result while $L e$ denotes either the endpoint of change on the main verb expressing Cause or the start point of change on Result. I thus assume that like DE in Chinese resultative constructions, the aspect marker $l e$ can also be indicated as a process. If so, the syntactic structure of the following sentence can be illustrated in (32), in which the null verb denoting a process can be replaced by the aspect marker $l e$.

(32) Ta ku-shi-le shoupa.

She cry-wet-ASP handkerchief

'She cried as a result that the handkerchief got wet.'

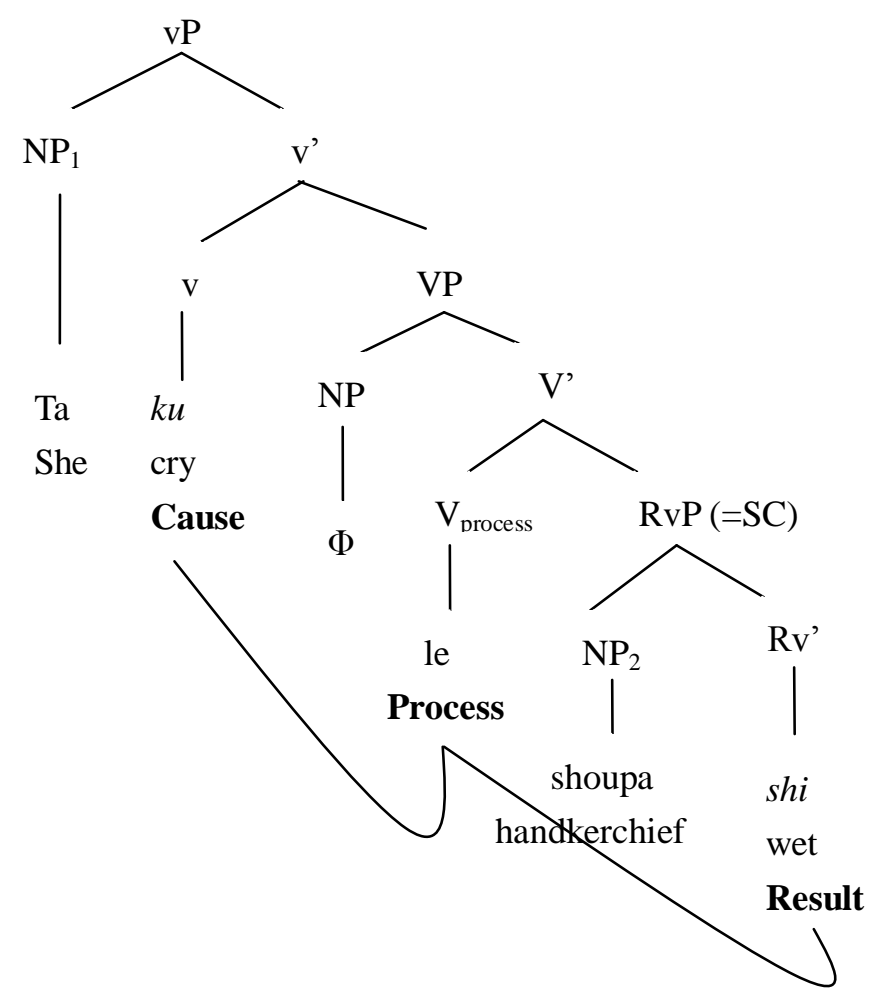

The structure (32) shows that the aspect marker $l e$ is indicated as a process. A bit different from DE in the V-DE-(NP)-V constructions, in (32), when le is the head of the SC shoupa shi 'the handkerchief wet', the SC is not a complete sentence, without tense, which leads $\mathrm{V}_{2} s h i$ 'wet' to be moved out to the place of le and then is moved to the place of the main verb together to compose the compound ku-shi-le 'cry-wet-ASP'.

Therefore, if both DE and the aspect marker le can be indicated as a process in Chinese resultative constructions, the ungrammaticality of weak V-DE-(NP)-A constructions can also be explained here. For example:

(33) $* \mathrm{Ta}$ tu de qiang bai-le.

He paint DE wall white-ASP

'He painted the wall white.' 


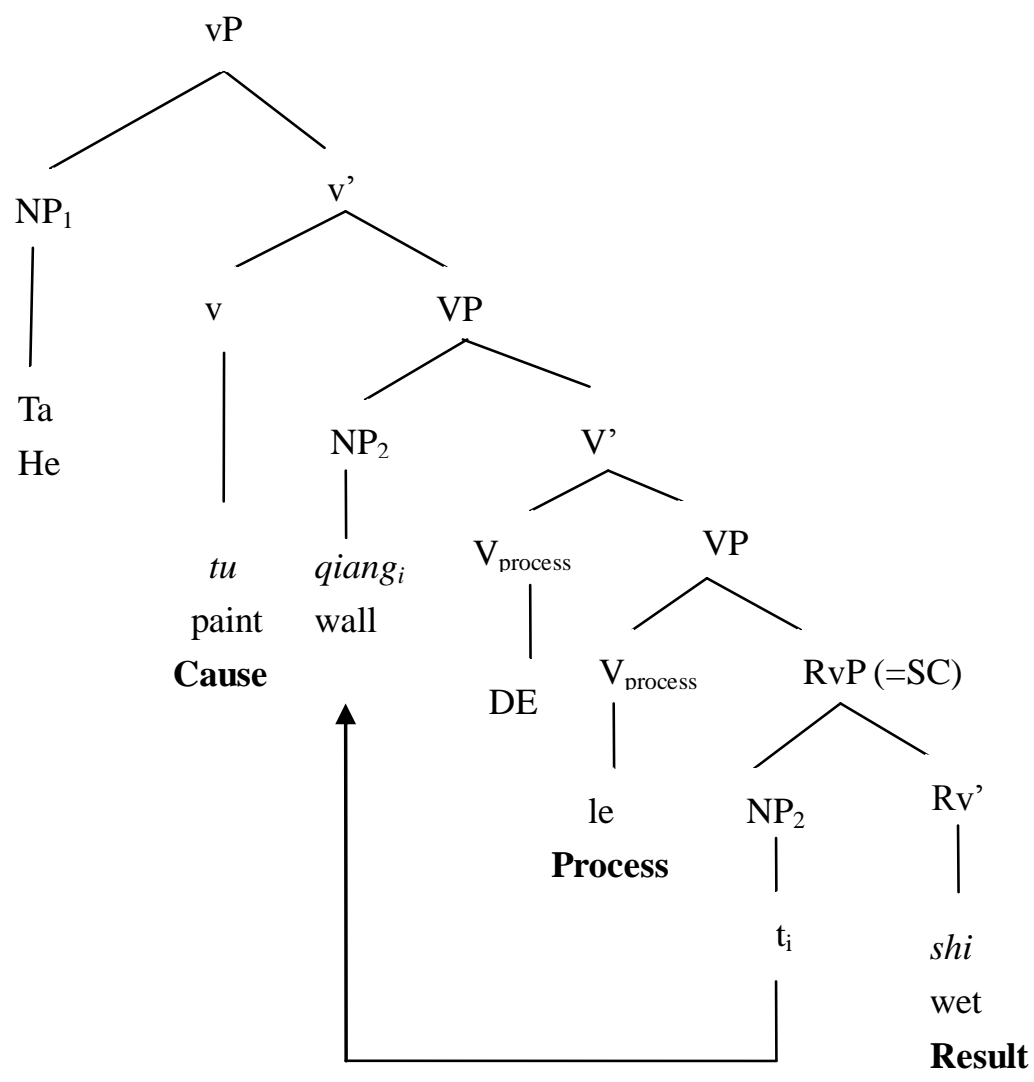

According to the assumption that the aspect marker le in Chinese resultatives is indicated as a process, in the structure of (33), it can be observed that there are two elements denoting a process, that is, DE and le. According to Folli (2001), as a Process, both DE and le can indicate change of state. Both DE and le are heads of the following SC, that is, SC has two heads in (33). In addition, the main verb $t u$ 'paint' entails the meaning of the result predicate bai 'white'. Therefore, the sentence (33) seriously violates the formation of a sentence in Chinese and so it must be ungrammatical.

\section{G. The Differences between DE and Le in Chinese Resultative Constructions}

It is illustrated that weak V-DE-(NP)-A constructions are ungrammatical in Chinese when the result predicate is the combination of a SLP + le, as in (34), but a fact is neglected that strong V-DE-(NP)-A constructions are grammatical in Chinese even if the result predicate is the SLP + le, as shown in (34).

$$
\begin{aligned}
& \text { (34) a. * John ran de qunzi hong-le. (weak) } \\
& \text { John dye DE dress red-ASP } \\
& \text { 'John dyed the dress red.' } \\
& \text { b. Ta } k u \text { de shoupa shi-le. (strong) } \\
& \text { She cry DE handkerchief wet-ASP } \\
& \text { 'She cried to an extent as a result the handkerchief got wet.' }
\end{aligned}
$$

Both DE and Le can be indicated as a Process, based on Folli and Lin in the paper, which denoting the change of state, i.e., semantically speaking, DE and Le can lexicalize the semantic element [BECOME]. In addition, in weak resultatives, it can be assumed that a covert verb, like DE or Le in V-DE-(NP)-A constructions, denotes a process, expressing a change of state, because of the definition of weak resultatives that the meaning of the main verb entails the meaning of the result predicate. In a short, in weak resultatives, a null verb can lexicalize the semantic element [BECOME]. If so, there seems to three semantic elements [BECOME] appearing in (34a). The sentence (34a) thus cannot be accepted. (34b), however, should be ungrammatical, because there are also two semantic elements [BECOME] appearing, i.e. $\mathrm{DE}=[\mathrm{BECOME}]$ and $\mathrm{Le}=[\mathrm{BECOME}]$, but in fact (34b) is acceptable. A question arises here as to how to explain the ungrammaticality of (34a) and the grammaticality of (34b). It can be realized that there is only one possibility that DE-le represents only one semantic element [BECOME]. In order to support this view, it is necessary here to distinguish $\mathrm{DE}$ with $l e$ and then discuss the possibility to combine them together to denote the semantic element [BECOME].

DE can serve as the process head in the syntactic structure of the V-DE-(NP)-A construction, proposed by Folli (2001) and Lin (2003), but I find that a fact that DE and Le co-occur in a sentence with restricted word orders, that is, DE always has to precede Le in the sentence. For example, the syntactic structure of the sentence (35) is the following tree 
diagram.

$\begin{array}{rrlll}\text { (35) } \mathrm{Ta} & \text { kan } & \mathrm{de} & \text { na-ben shu } & \text { lan-le. } \\ \mathrm{He} & \text { read } & \mathrm{DE} & \text { that book } & \text { broken-ASP }\end{array}$

'He read that book as a result that it was broken.'

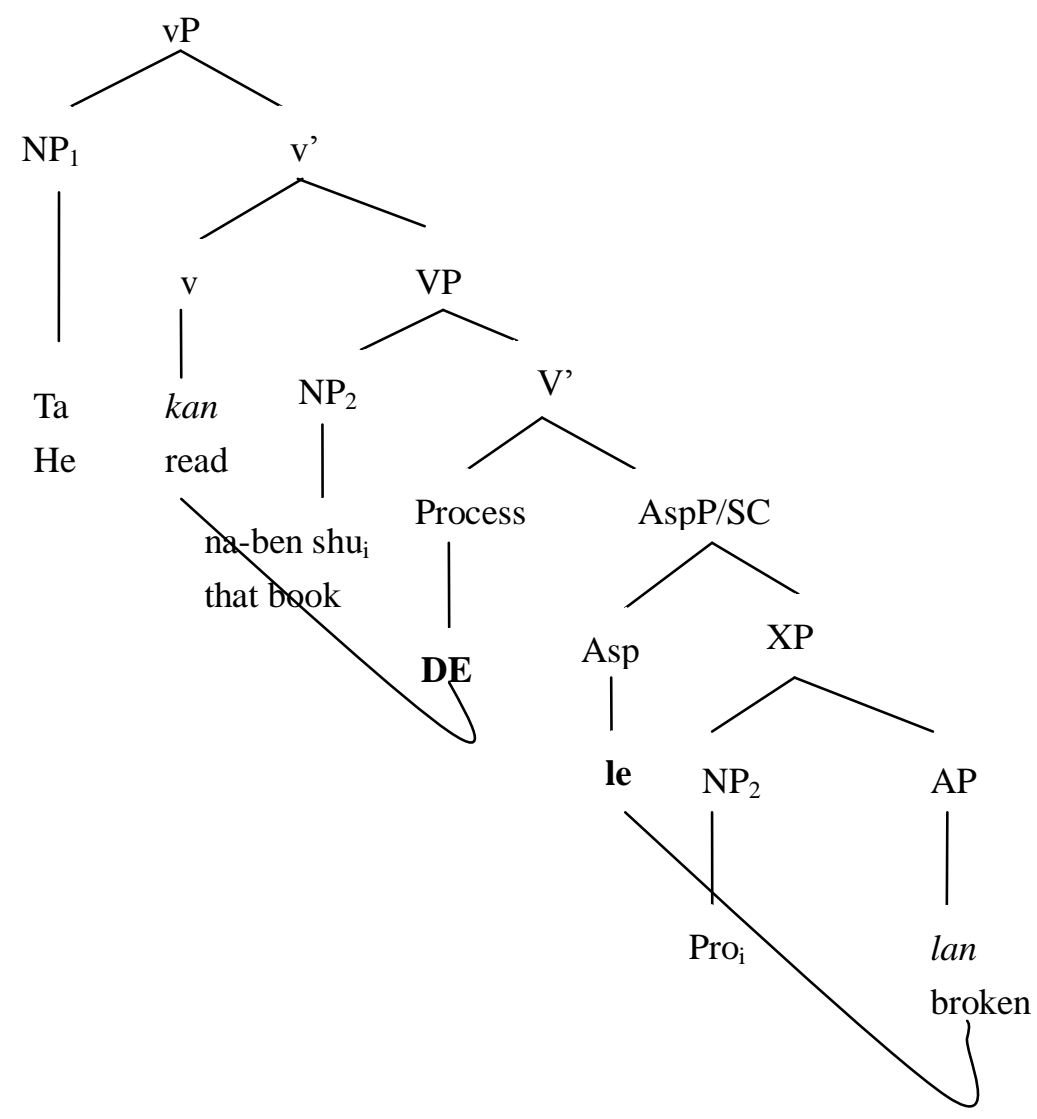

In (35), it can be found that DE, as a process head, occurs at a higher position than Le in Chinese V-DE-(NP)-A constructions, which shows a fact that Le cannot denote a process when DE appears in the sentence. On the other hand, in the SC, the result predicate SLP can be moved to the place of Le to compose the compound SLP+le, but this compound cannot be continuously moved to the place of the main verb, because of the block of DE. Thus, only DE can be moved to the place of the main verb.

It is certain that the sentence is ungrammatical when Le precedes DE, as in (36).
(36) a. *Ta kan le de na-ben shu lan.
He read ASP DE that book broken
b. *Ta kan le na-ben shu lan de.
He read ASP that book broken DE

'He read that book as a result that it was broken.'

The example (36) testifies to the assumption that Le cannot be indicated as a process in Chinese resultative construction.

To sum up, DE can be denoted as a semantic element [BECOME] in the V-DE-(NP)-A construction in semantics. In addition, I also argue that the aspect marker Le can also lexicalize the semantic element [BECOME] in semantics. Although both DE and Le can lexicalize the semantic element [BECOME], indicating the change of state, it can be realized that DE indicates the action denoted by the main verb has arrived at a state but this action is not completed, whereas Le can indicate the endpoint of the action but not be denoted as a process. For example:

$$
\begin{array}{rrrrr}
\text { (37) a. * Ta } & k u & \text { de } & \text { shoupa } & \text { shi. } \\
\text { He } & \text { cry } & \text { DE handkerchief } & \text { wet } \\
\text { b. Ta } & k u & \text { de } & \text { shoupa } & \text { shi-le. } \\
\text { He } & \text { cry } & \text { DE handkerchief } & \text { wet-ASP }
\end{array}
$$

'He cried as a result that the handkerchief got wet.'

In (37), the lack of $L e$ leads to the sentence ungrammatical even if DE appears in the sentence, which shows that only when both DE and Le appear in the sentence, the sentence is grammatical. Thus, it can be assumed that DE cannot be separated from $L e$ when we want to express the change of the state and the endpoint of the action in a Chinese resultative construction at the same time, that is, only DE or Le cannot completely indicate the action denoted by the 
main verb has arrived at a state and this action has been completed. If so, the ungrammaticality of weak V-DE-(NP)-A constructions can be explained now. Although weak V-DE-(NP)-A constructions are ungrammatical in Chinese, when the result predicate is an ILP or is modified by a degree word ungrammatical weak V-DE-(NP)-A constructions can be acceptable. Why so? Section 4 will focus on this question.

\section{The Possibility of GRAmmatical WeAK V-DE-(NP)-A CONSTRUCTIONS IN Chinese}

In section 3, under the assumptions that both DE and Le in Chinese resultative constructions can be indicated as a process, the question is accounted for why weak V-DE-(NP)-A constructions are ungrammatical. In fact, when the result predicate is an ILP or is modified as a degree word in weak V-DE-(NP)-A constructions, it can be found that ungrammatical weak ones can be acceptable in Chinese. Consider a pair of sentences in (38) at first.

$$
\begin{aligned}
& \text { (38) a. * Ta tu de qiang bai-le. } \\
& \text { He paint DE wall white-ASP } \\
& \text { 'He painted the wall white.' } \\
& \text { b. Ta tu de qiang xue-bai. } \\
& \text { He paint DE wall snow-white } \\
& \text { 'He painted the wall snow-white.' } \\
& \text { c. Ta tu de qiang tebie } \\
& \text { He paint DE wai. } \\
& \text { 'He painted the wall especially white.' }
\end{aligned}
$$

(38a) is ungrammatical when the result predicate is the combination of a SLP+Le, i.e., bai-le 'white-ASP', whereas the sentence becomes grammatical when the result predicate is an ILP xue-bai 'snow-white' in (38b). And when the result predicate is the combination of a degree word + SLP, i.e., tebie white 'especially white', the sentence (38c) also becomes acceptable in Chinese. At first, I will account for the question why ungrammatical weak V-DE-(NP)-A constructions are acceptable when the result predicate is an ILP in section 4.3.

\section{A. The Possibility of Grammatical Weak V-DE-(NP)-A: On the Occasion of ILP}

Consider a pair of sentences in (39) at first:

$$
\begin{aligned}
& \text { (39) a. * Ta tu de qiang bai-le. } \\
& \text { He paint DE wall white-ASP } \\
& \text { 'He painted the wall white.' } \\
& \text { b. Ta tu de qiang xue-bai } \\
& \mathrm{He} \text { paint DE wall snow-white } \\
& \text { 'He painted the wall snow-white.' }
\end{aligned}
$$

In (39a), in weak resultatives, I repeat, the meaning of the main verb entails the meaning of the result predicate. Thus, it is assumed that there is an originally covert semantic element [BECOME] in weak resultatives. If DE-le appears in weak resultatives, the sentence will be ungrammatical, as in (39a), because DE-le can indicate a complete semantic element [BECOME] in Chinese resultative constructions. In (39b), the result predicate is an ILP xue-bai 'snow-white' not a SLP+le and the sentence is grammatical. I argue that the ILP indicates the state and DE-ILP, i.e. DE-State cannot be denoted as a semantic element [BECOME], while the covert semantic element [BECOME] is implied in weak resultatives, the sentence (39b) thus is grammatical.

The syntactic structure of the ungrammatical weak V-DE-(NP)-A construction has been discussed, like (39a) in section 3, that is, the syntactic structures of weak resultative V-A-(NP) compounds can be illustrated in terms of two-layer system (Cause and Result). When DE, as a process, appears, it can obstruct the connection of the meanings of the main verb and the result predicate. Thus, weak V-DE-(NP)-A constructions are ungrammatical in Chinese. As to the syntactic structure of (39b), I will give my own analysis here. (39b) is still a weak resultative, in which the meaning of the main verb $t u$ 'paint' implies the meaning of the result predicate xue-bai 'snow-white'. The syntactic structure of (39b) thus can be analyzed based on my two-layer-system proposal. However, DE is allowed in such sentences. A question arises as to how to deal with DE in (39b). I suggest a reason for this question. It is assumed that when the result predicate is an ILP in weak V-DE-(NP)-A constructions, two properties of DE are restricted, that is, as a process and as a semantic element [BECOME], indicating change of state. And I assume that DE here appears to be a verbal suffix, which means that DE can be combined with the main verb to compose a compound V-de. If so, depending on the two-layer system, the syntactic structure of (39b) can be illustrated in (40).
(40) Ta tu-de qiang xue-bai.
He paint-DE wall snow-white 


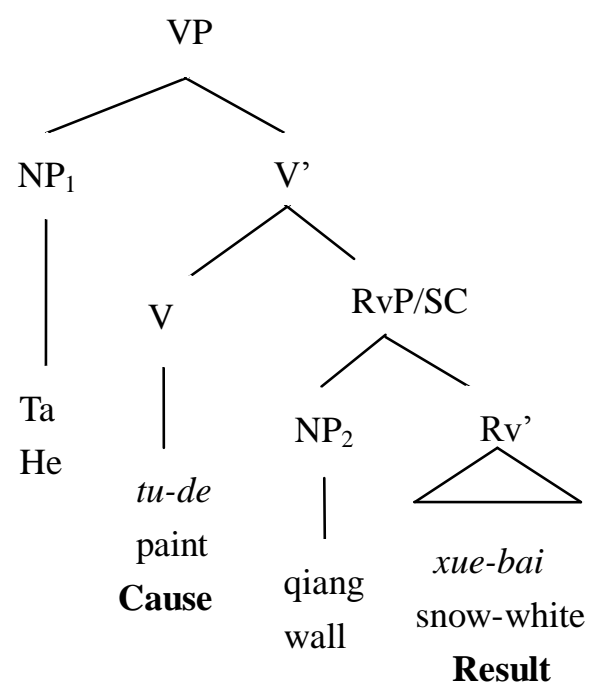

The syntactic structure of (40) shows that DE, as a verbal suffix, following the main verb, is more reasonable than as a head of the SC, followed by the result predicate when it is analyzed from the perspective of the two-layer system. If $\mathrm{DE}$ is indicated as the head of the SC, the syntactic structure of (40) will be illustrated as in (41).

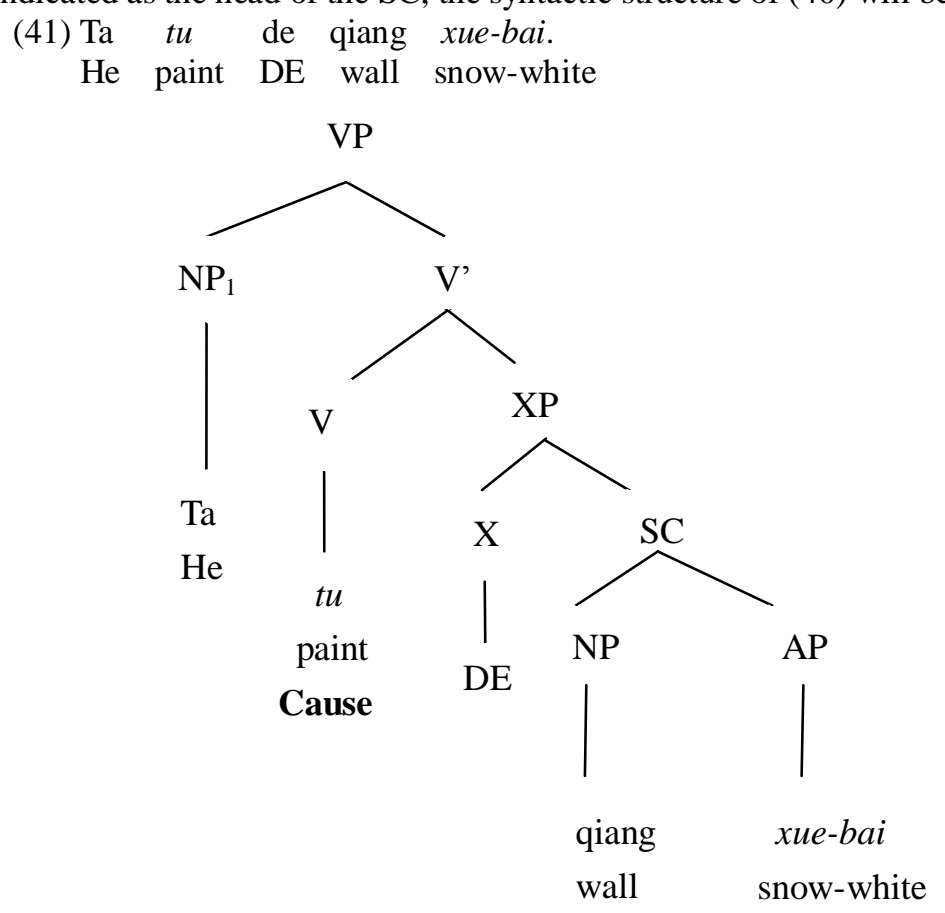

Result

In (41), if DE, as the head of the SC, is followed by the SC, the two-layer system (Cause and Result) will be against, because DE adds a new subevent $X$ in the syntactic structure. Compared with (40), (41) is untenable. Thus, the reasonable syntactic structure of the weak V-DE-(NP)-A construction is shown in (40), in which the result predicate is an ILP.

B. The Possibility of Grammatical Weak V-DE-(NP)-A: On the Occasion of Degree Words

There is another possibility to make ungrammatical weak V-DE-(NP)-A constructions acceptable, that is, when the result predicate is modified by a degree (Deg) word. For example:
(42) a. ${ }^{*} \mathrm{Ta}$ tu de qiang bai-le.
He paint DE wall white-ASP
'He painted the wall white.'
b. Ta tu de qiang hen bai.
He paint DE wall very white
'He painted the wall very white.'


In (42b), it can be found that when the result predicate SLP + le is modified by the degree word hen 'very', the aspect marker le disappears. Why so? I claim that $l e$ is the head of the combination of SLP + le, which can be indicated as the semantic element [BECOME]. When the degree word modifies the compound SLP + le, only the SLP is modified by the degree word but not le, namely, hen [bai-le] is equal to very [become white] in English and it is vivid that the degree word very cannot modify the verb become in English. This situation is the same in Chinese. Thus, the aspect marker, denoting the change of state, must be deleted when the degree word appears, as in (42b).

Consideration of the example (42b) again, a question arises as to why the sentence (42b) is grammatical. It is realized that this question must be related to the property of the combination of Deg + SLP. I argue here that when the SLP is combined with the degree word, the property of the SLP is lost, like denoting transitory and accidental properties. The combination of Deg-SLP can function as an ILP, denoting permanent and essential properties. For example:
(43) a. Shui liang-le, keyi he le.
Water cold-ASP, can drink le
'The water has been cold and can drink.'
b. Shui hen liang, buneng he.
'The water is very cold and cannot drink.'

In (43), the SLP liang 'cold' is ambiguous. When it combines with the time-phased auxiliary le, a compound liang-le 'cold+ASP' can be formed, which means BECOME COLD in Chinese, as in (43a). The sentence shui liang le 'the water has been cold' describes the temperature change of water and expresses the complete event structure. In (43b), when only the SLP liang 'cold' is modified by the degree word hen 'very', hen liang 'very cold' can be interpreted as an ILP, which means that the semantic element [BECOME] cannot be allowed. The sentence shui hen liang 'the water is very cold' describes the temperature property of the water and expresses the static event structure. Thus, it can be said that the appearance of the degree word hen 'very' can suppress the semantic element [BECOME] and conversely highlight the semantic element [BE], as an ILP, as in (43b). More one simple example is given in (44) in Chinese.
(44) a. Ta mei le.
She beautiful ASP
'She becomes beautiful.'
b. Ta hen mei.
She very beautiful
'She is very beautiful.'

In (44a), the combination $m e i+l e$ 'beautiful+ASP' in Chinese means BECOME BEAUTIFUL in English. When the degree word hen 'very' modifies the compound mei+le 'beautiful+ASP', it only modifies the SLP mei 'beautiful', as in (44b), the combination of hen $m e i$ 'very beautiful' can function as an ILP to express permanent or inherent properties. If so, I propose that the syntactic structure of the weak V-DE-(NP)-Deg + SLP construction is the same as one of the weak V-DE-(NP)-ILP construction, as shown in (45).
(45) a. Ta tu-de qiang $\underline{\text { hen }}$ bai.
He paint-DE wall very white
'He painted the wall very white.'

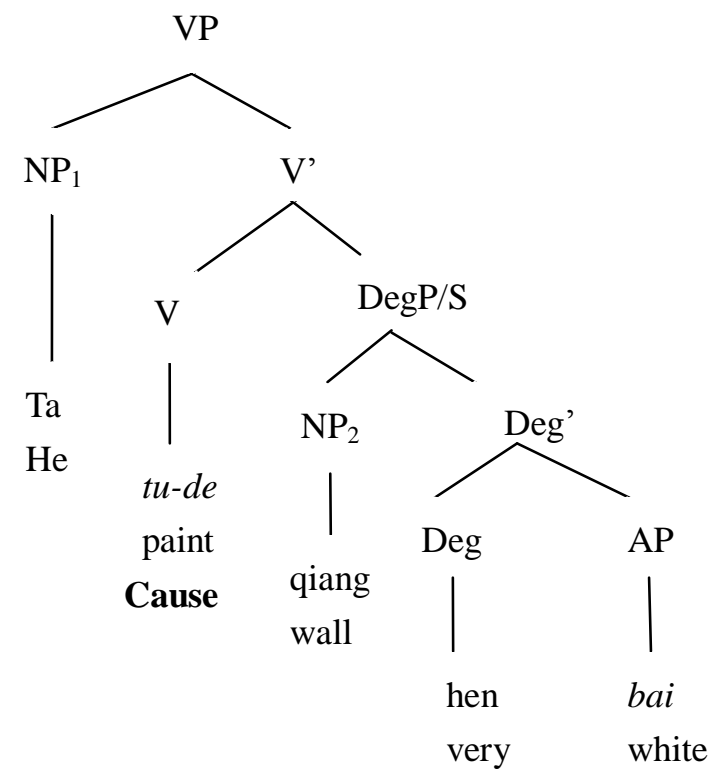

Result 
In (45), because the combination of Deg-SLP can function as an ILP, the syntactic structure of the V-DE-(NP)-Deg + SLP can be illustrated in terms of the two-layer system, in which Cause is denoted by the combination of the main verb $+\mathrm{DE}$ and Result is expressed by the combination of Deg + SLP.

To sum up, as to ungrammatical weak V-DE-(NP)-A constructions, an interesting linguistic phenomenon shows that these ungrammatical weak V-DE-(NP)-A constructions can be acceptable in Chinese when the result predicate is an ILP or is modified by the degree word. Why so? I suggest a reason for this question in this section, that is, DE-SLP + le $=$ $[\mathrm{BECOME}], \mathrm{ILP}=[\mathrm{STATE}]$ and Deg-SLP $=$ [STATE $]$ and then DE-ILP $\neq$ [BECOME], DE-Deg-SLP $\neq$ [BECOME]. Based on these formulas, it can be said that ungrammatical weak V-DE-(NP)-A constructions are possible to be acceptable in Chinese.

\section{CONCLUSION}

The paper focuses on discussing the possible weak V-DE-(NP)-A constructions in Chinese resultative constructions, when the result predicate is an ILP or is modified by a degree word. In general, when the result predicate is the combination of a SLP + le, weak V-DE-(NP)-A constructions are ungrammatical, because both DE and Le can function as a Process, denoting a change of state, which can be indicated as a semantic element [BECOME] in Chinese resultative constructions. In addition, the meaning of the main verb entails the meaning of the result predicate in weak resultatives, so there is a covert semantic element [BECOME] in these constructions. However, when the result predicate becomes an ILP or a degree word + SLP, weak V-DE-(NP)-A constructions become grammatical, which is related to the property of ILP and Deg+SLP, opposing to the property of SLP+le. Because DE-ILP and DE-Deg+SLP cannot lexicalize the semantic element [BECOME] and a covert semantic element [BECOME] is allowed in a weak resultative because of the entailment of the meanings of the main verb and the result predicate. Therefore, weak V-DE-(NP)-A constructions become grammatical when the result predicate is an ILP or is modified by a degree word.

\section{REFERENCES}

[1] Carlson, Gregory N. (1977). Reference to Kinds in English. PhD dissertation. UMass/Amherst.

[2] Cheng, Lisa Lai-Shen, and Huang, C.- T. James. (1994). On the argument structure of resultative compounds. In Honor of William S-Y. Wang: Interdisciplinary Studies on Language and Language Change, ed. by Matthew Y. Chen and Ovid J. L. Tzeng, 187-221.Taipei: Pyramid Press.

[3] Davidson, D. (1967). The Logical Form of Action Sentences. In The Logical of Decision and Action, N.Rescher, ed. Pittsburgh: University of Pittsburgh Press. 105-121.

[4] Ding, Shengshu. (1961). Modern Chinese Grammar Speech. Commercial Press, Beijing.

[5] Folli, Raffaella. (2001). Constructing Telicity in English and Italian. Ph.D. thesis, Oxford University.

[6] Kratzer, Angelika. (1995). Stage-level and Individual-level Predicates. In Gregory N. Carlson and Francis J. Pelletier (eds), The Generic Book. University of Chicago Press. 125-175.

[7] Li,Yafei. (1998). Chinese resultative constructions and the uniformity of theta assignment hypothesis. In New Approaches to Chinese Word Formation: Morphology, Phonology and the Lexicon in Modern and Ancient Chinese, ed. by Jerome L. Packard, 285-310. New York:Mouton de Gruyter.

[8] Li, Yafei. (2003). Localizers in Chinese and the Cost of Computation. Ms. University of Wisconsin, Madison.

[9] Lin, Chienjer. (2003). Aspect is result: Mandarin resultative constructions and aspect incorporation. Proceedings of the Western Conference on Linguistics 32.437-464.

[10] Washio, Ryuichi. (1997). Resultatives, compositionality and language variation.Journal of EastAsianLinguistics 6, 1-49.

[11] Zhang Guoxian. (1995). Dynamic adjectives in modern Chinese. Chinese Language, $3^{\text {rd }}$ Issue, 221-229.

[12] Zhu Dexi. (1982). Graduation Lecture. Commercial Press, Beijing.

Xiaowen Zhang, female, Ph.D. degree of Tokyo Gakugei University, focusing on comparative linguistics, including English, Japanese and Chinese, a lecturer, Foreign Language Department of Huaiyin Institute of Technology, Huaian of Jiangsu Province, China. This paper serves for the Program of Philosophy and Social Science Research in Colleges and Universities of Jiangsu Province (Number: 2019SJA1652; Title: A Contrastive Study of Resultative Constructions in Chinese, Japanese and English). 